\title{
Inappropriate assumptions about EEG state changes and their impact on the quantification of EEG state dynamics
}

Comment on:

M Gärtner, V Brodbeck, H Laufs, G Schneider: A stochastic model for EEG microstate sequence analysis. Neurolmage 104 (2015) 199-208

Thomas Koenig ${ }^{1}$, and Daniel Brandeis ${ }^{2,3}$

1) Psychiatric Electrophysiology Unit

Translational Research Center University Hospital of Psychiatry University of Bern, Switzerland Tel: ++41 0(31) 930 9369, Fax: ++41 0(31) 9309961 mail: thomas.koenig@puk.unibe.ch

2) Brain Mapping Department of Child and Adolescent Psychiatry University of Zürich, Switzerland

3) Developmental Clinical Neurophysiology Department of Child and Adolescent Psychiatry and Psychotherapy Central Institute of Mental Health Medical Faculty Mannheim /Heidelberg University, Germany

Words: 1942 (including title, figure legends and references), 2 figures

Keywords: Microstates, Global Field Power, Topographic Change 


\section{Dear Editor,}

It was with great interest that we read the recently published paper by Gärtner and colleagues (Gärtner et al., 2015) in Neuroimage regarding EEG microstates and background processes. The parcellation of multichannel continuous EEG data into periods of quasi-stable spatial configuration of oscillatory activity (microstate analysis) is becoming increasingly recognized as an innovative analysis for the spontaneous organization of brain function, and has frequently been covered by this journal (Britz et al., 2010; Brodbeck et al., 2012; Custo et al., 2014; Gärtner et al., 2015; Katayama et al., 2007; Koenig et al., 2002; Musso et al., 2010; Yuan et al., 2012). This interest is timely, because conceptually very similar approaches (i.e., focusing on temporally coherent network activity) have become a powerful tool to analyze fMRI resting state data.

The paper by Gärtner and colleagues addresses an important technical issue in the analysis of transiently stable EEG microstates. Since there is predominantly oscillatory activity in EEG, the signal is small and noisy around the moments of polarity reversal of the EEG field, which complicates the attribution of those EEG spatial configurations to a particular microstate. Many EEG studies have dealt with this problem by analyzing and attributing only time-points where the EEG field amplitude has a momentary maximum (the so-called Global Field Power (GFP) peak), and interpolating the assignment of these periods in between those maxima, thereby addressing the topographic stability across GFP peaks. These studies have quite consistently yielded microstate durations of approximately 60-100 msec, assuming 4 classes of microstates (Koenig et al., 2002).

Gärtner and colleagues rightfully criticize the above described methodology for being incomplete, since a considerable part of the results is based on interpolation. Given that an EEG is dominated by alphaband activity, one would expect around 20 GFP peaks per second, which at a standard sampling rate of $250 \mathrm{~Hz}$, amounts to less than $10 \%$ of the data points. They propose a theoretical model that assumes that the sequence of EEG microstates may be explained as the result of a sparse sampling of an unobservable underlying background process. Based on the assumptions discussed in more detail below, but still relying only on observations made at GFP peaks, they estimate the duration of the states of this background process to be around $10 \mathrm{msec}$, which is much shorter than what the literature has reported on microstates. Although their model still reproduces longer microstate durations when considering only the stability across GFP peaks, it implies that the observation that microstate durations are much longer than state durations of the background process is a result of the sparse sampling of the background process. 
Such a major difference of a basic state property obtained using different models calls for further explanation. Has the initial methodology used for EEG microstates drastically oversimplified the processes that it should be accounting for? Can the differences be accounted for by the methods, which are measuring something different but complementary in nature, such as the microstate stability across GFP peaks versus the more subtle transitions between background intervals within these GFP peak microstates, possibly also at different frequencies? Or is there a problem with the methodology proposed here? We completely agree that the traditional microstate methodology could systematically overestimate mean state duration due to the sparse sampling at GFP peaks. However, mean durations of approximately $80 \mathrm{msec}$ were identified in recent EEG papers where microstates have been fitted to the entire data set (Tomescu et al., 2014).

A core assumption of the methods proposed by Gärtner and colleagues is not supported by the available empirical data. The authors estimate the duration of unobservable brain states and transitions of a hypothetical background process using sparsely sampled data at GFP peaks. In order to do this, 2 assumptions were introduced, namely that a) the background process can be described as a Poisson process, and that $b$ ) the sampling of the state (i.e. the moments of a local maximum of the GFP) is "an independent renewal process of random sampling points" and thus, independent from the probability of a transition between states. Using these assumptions and taking into account the given observations of momentary state at the GFP maxima, the rate of state transitions and the duration of the states (as defined by the mostly unobserved background process) are then estimated, based on the topographies observed at GFP peaks (Gärtner et al., 2015, page 203).

The assumption that the occurrence of GFP peaks is independent of topographic change is counterfactual. In the models being discussed here, the state is defined by a presumably constant topography plus noise; therefore, the probability of a state transition is a function of topographic change per time, which is empirically found to be strongly dependent on GFP. One can quantify the speed of this topographic change (e.g. by using 1 minus the percent of common variance between adjacent maps). This unit-less measure is mathematically directly related to the computation of spatial dissimilarity (Lehmann and Skrandies, 1980), but is more informative in the current context. A plot of momentary topographic change against momentary GFP reveals that moments of high GFP values invariably coincide with very little topographic change, whereas moments of low GFP may or may not exhibit large topographic changes (Fig. 1). The assumption of independence between the occurrence of GFP peaks and topographic change can therefore not be maintained. It is important to note that the 
percent common variance between the maps used here to quantify topographic change is also used to assign momentary maps to particular states, both in microstate methodology and in the Gärtner model.

The authors have rightfully argued why peaks of topographic change (peaks of dissimilarity), which are often in between GFP peaks, are not suitable to define state transitions. However, this does imply that the values of momentary topographic change or dissimilarity as function of time are not informative for state stability and can be dismissed. Despite the fact that the background process itself is unobservable, we can use available information regarding momentary topographic change to test predictions made by the authors' model. If we assume the mean interval for the background process is $10 \mathrm{msec}$ and compute the amount of topographic change between the time points that are $10 \mathrm{msec}$ apart across all samples, one should obtain a large amount of values (about $50 \%$ ) that are in a range that qualifies for a change in state labeling, according to the estimated background process. However, as Fig. 2 shows, this is not the case. In the microstate prototype maps from Koenig et al. (2002), the topographic change between state classes ranges from 0.36 to 0.99 . Even when assigning the topographic change between the 2 most similar microstate prototype maps as the threshold, only about $7 \%$ of all topographic changes of maps that are 10 msec apart reveal changes that are larger than this threshold, and would therefore qualify as state transitions. This point makes it evident that the authors' initial assumptions, and therefore their results and derived conclusions, are far from being compatible with available empirical data.

The conclusion we want to communicate here is that the assumptions made by the proposed method are partially incompatible with the basic and empirically evident features of EEG data and thus, need to be adapted. Such an adapted approach should not only use valid assumptions, but also attempt to validate hypothetical background states that are only "unobservable" under sparse sampling by taking the actual EEG data between GFP peaks into account. This may be achieved by integrating the empirically available relationship between topographic changes and GFP into the model, and fitting microstate topographies across the entire data (Tomescu et al., 2014). In addition, the application of some reasonably justified filters would reduce the problem of noisy assignments during polarity reversals (Pascual-Marqui et al., 1995; Van de Ville et al., 2010). Alternatively, using methods that can define the topography during polarity reversals using complex basis functions would also be appropriate (Koenig et al., 2001). 


\section{References}

Britz, J., Van De Ville, D., Michel, C.M., 2010. BOLD correlates of EEG topography reveal rapid restingstate network dynamics. Neuroimage 52, 1162-1170.

Brodbeck, V., Kuhn, A., von Wegner, F., Morzelewski, A., Tagliazucchi, E., Borisov, S., Michel, C.M., Laufs, H., 2012. EEG microstates of wakefulness and NREM sleep. Neuroimage 62, 2129-2139.

Custo, A., Vulliemoz, S., Grouiller, F., Van De Ville, D., Michel, C., 2014. EEG source imaging of brain states using spatiotemporal regression. Neuroimage 96, 106-116.

Gärtner, M., Brodbeck, V., Laufs, H., Schneider, G., 2015. A stochastic model for EEG microstate sequence analysis. Neuroimage 104, 199-208.

Katayama, H., Gianotti, L.R., Isotani, T., Faber, P.L., Sasada, K., Kinoshita, T., Lehmann, D., 2007. Classes of multichannel EEG microstates in light and deep hypnotic conditions. Brain Topogr 20, 7-14.

Koenig, T., Marti-Lopez, F., Valdes-Sosa, P., 2001. Topographic time-frequency decomposition of the EEG. Neuroimage 14, 383-390.

Koenig, T., Prichep, L., Lehmann, D., Sosa, P.V., Braeker, E., Kleinlogel, H., Isenhart, R., John, E.R., 2002. Millisecond by millisecond, year by year: normative EEG microstates and developmental stages. Neuroimage 16, 41-48.

Lehmann, D., Skrandies, W., 1980. Reference-free identification of components of checkerboard-evoked multichannel potential fields. Electroencephalogr Clin Neurophysiol 48, 609-621.

Musso, F., Brinkmeyer, J., Mobascher, A., Warbrick, T., Winterer, G., 2010. Spontaneous brain activity and EEG microstates. A novel EEG/fMRI analysis approach to explore resting-state networks. Neuroimage 52, 1149-1161.

Pascual-Marqui, R.D., Michel, C.M., Lehmann, D., 1995. Segmentation of brain electrical activity into microstates: model estimation and validation. IEEE Trans Biomed Eng 42, 658-665.

Tomescu, M.I., Rihs, T.A., Becker, R., Britz, J., Custo, A., Grouiller, F., Schneider, M., Debbane, M., Eliez, S., Michel, C.M., 2014. Deviant dynamics of EEG resting state pattern in 22q11.2 deletion syndrome adolescents: A vulnerability marker of schizophrenia? Schizophr Res 157, 175-181.

Van de Ville, D., Britz, J., Michel, C.M., 2010. EEG microstate sequences in healthy humans at rest reveal scale-free dynamics. Proc Natl Acad Sci U S A 107, 18179-18184.

Yuan, H., Zotev, V., Phillips, R., Drevets, W.C., Bodurka, J., 2012. Spatiotemporal dynamics of the brain at rest--exploring EEG microstates as electrophysiological signatures of BOLD resting state networks. Neuroimage 60, 2062-2072. 
Figure legends:

Fig. 1: Momentary GFP against topographic change between adjacent maps (1 - percent common variance) in a randomly selected awake, eyes-closed 70 channel EEG $1 \mathrm{~h}$ in length. Upper rows: observed data; lower rows: same data, but after separately shuffling the $x$ (GFP) and y (topographic change) values to simulate the assumptions by Gärtner and colleagues. Left column; untransformed data; right column, after log-log transformation. After log-log transformation, the two values correlate with an $r$ of .725 , which indicates a common variance above $50 \%$. This is opposed to the model by Gärtner, which assumes independence.

Fig. 2 Black line: The probability that the topographic change between any two maps 10 msec apart reaches a certain threshold, obtained from the same data as used for Fig.1. These thresholds are defined by the empirically observed topographic changes between a given set of microstate prototype maps. Dotted lines: The thresholds for state changes, as obtained from the microstate prototype maps obtained from a large normative database. Even for the smallest threshold, which corresponds to the transitions between the two most spatially similar microstate prototype maps, the probability is only $7 \%$, which is incompatible with a mean state duration of $10 \mathrm{msec}$. 